\title{
On Hemostasis of Cold Atmospheric Air Plasma
}

\author{
Spencer Kuo \\ Department of Electrical \& Computer Engineering, New York University-Tandon School of Engineering, Brooklyn, NY, USA \\ Email: spk259@nyu.edu
}

How to cite this paper: Kuo, S. (2018) On Hemostasis of Cold Atmospheric Air Plasma. Open Journal of Emergency Medicine, 6, 99-111. https://doi.org/10.4236/ojem.2018.64012

Received: October 25, 2018

Accepted: December 11, 2018

Published: December 14, 2018

Copyright (c) 2018 by author and Scientific Research Publishing Inc. This work is licensed under the Creative Commons Attribution International License (CC BY 4.0).

http://creativecommons.org/licenses/by/4.0/

\begin{abstract}
The efficacy and mechanism of a cold atmospheric-pressure air plasma (CAAP), which carries abundant atomic oxygen (OI), on blood coagulation are studied. The tests on sodium citrate mixed blood-droplet samples show that 1) The heat delivered by the CAAP has no impact on the observed clot formation, 2) Plasma effluent activates platelets to promote coagulation state and cascade, and 3) The degree of clotting increases with the OI flux delivered by the CAAP. The full clotting time is shortened from about 25 minutes of the natural clotting time to about $16 \mathrm{~s}$ of the CAAP treatment time. The tests on smeared blood samples show that the reduction of the platelet count and the increase of RBC count are proportional to the applied OI flux. In vivo tests, using swine as animal model, swift hemostasis of large and deep cut wounds on the back by the CAAP treatment was demonstrated. A cut artery was sealed completely with $25 \mathrm{~s}$ treatment. The pressure applied by a finger on the cut artery could be removed immediately after the treatment and there was no re-bleed. Based on the in vitro test results and the animal model trials, CAAP coagulation mechanism is presented.
\end{abstract}

\section{Keywords}

Cold Atmospheric Air Plasma, Cold Atmospheric Plasma, Reactive Species, Reactive Oxygen Species, Atomic Oxygen, Trauma, Hemorrhage,

Hemostasis, Swine

\section{Introduction}

Atmospheric pressure plasmas, used in medical applications, can be generated by various electric discharges [1] [2] [3] [4]; however, plasmas have to be generated in a non-equilibrium state, so that the electron kinetic energy, gained from the electric source, is better used for producing reactive species, rather than for heating the plasma effluent; keeping plasma in low temperature. The reactive species carried by the plasma effluent can trigger biochemical reactions, which 
furnish biocidal effects for sterilization applications, speed up coagulation cascade for hemostasis applications, and promote wound healing [5]-[15].

The flux of the reactive species carried by the plasma is somehow proportional to the discharge current, the concentration of $\mathrm{O}_{2} / \mathrm{N}_{2}$ in the working gas, and the percentage of the energetic electrons in the electron plasma. Noble gases and noble gas mixed with $\mathrm{N}_{2} / \mathrm{O}_{2}$ have been used as a way to lower the plasma temperature [2] [4] [5] [6], but the tradeoff lowers the intensities of generated reactive species.

On the other hand, using ambient air as the working gas, chemically reactive oxygen species (ROS) and nitrogen species (RNS), including molecular oxygen/nitrogen in metastable states and atomic oxygen/nitrogen, are generated directly in the plasma. Air flow and/or magnetic field have been introduced in devices to generate cold atmospheric air plasma (CAAP) spray [16], where the discharge paths are elongated by the airflow, and rotating in the magnetic field to keep electric discharge diffusive. In the other words, the arc column dragged by the gas flow evolves to gliding arc mode. The non-equilibrium stage starts when the length of the gliding arc exceeds a critical value. The magnetic field rotates the gliding arc, making it diffusive and prolonging the non-equilibrium state. A periodic voltage source also works to maintain the non-equilibrium state. The advantage of running the air discharge in low voltage high current diffusive gliding arc mode is that the power supply can be compact and additional gas tank is not necessary, which make easy to design a practical portable CAAP device [16].

The present work explores the blood coagulation mechanism of a CAAP [16] via in-vitro tests on blood-droplet samples and on smeared blood samples, as well as via in-vivo trial, using swine as animal model. The efficacy of this CAAP in rapid control of active hemorrhage is demonstrated. The test results are presented and discussed.

\section{In-Vitro Tests to Explore Plasma Coagulation Mechanism}

Blood is a fluid tissue that includes $60 \%$ of a liquid portion known as blood plasma, and $40 \%$ of formed elements or blood cells [17].

Both formed elements and blood plasma contribute to blood coagulation during hemorrhage. Blood coagulation involves platelet activation and coagulation cascade. When the platelets encounter the break situation of the blood vessel, molecules touching the platelets trigger platelet activation; it is followed by the coagulation cascade stepping up blood clotting. Several proteins (fibrinogen, tissue factor, calcium, etc.) and molecules, called coagulation factors, play important roles in the coagulation cascade.

Cold atmospheric air plasma (CAAP) carries free radicals, such as reactive oxygen species (ROS), can quickly stop wound bleeding [12] [13]; a study of the air plasma coagulation mechanism, based on the tests employing a CAAP [16] to treat the blood droplet samples and on the smeared blood samples, is presented 
in the following. Each test with a specific treatment time (i.e., from 2 to $16 \mathrm{~s}$ ) and at a specific exposure distance (i.e., $25 \mathrm{~mm}$ or $40 \mathrm{~mm}$ ) was repeated once. The emission spectroscopy of the plasma effluent indicates that the dominant ROS in this CAAP is atomic oxygen (OI) [18].

Blood samples used in tests were mixed with 3.2\% sodium citrate solution at 9:1 ratio (in volume). The sodium citrate solution chelates calcium ions to prolong the natural clotting time [19] [20] to more than 25 minutes. Because Formed elements and blood plasma involve in different ways in contributing blood coagulation during hemorrhage; they should be separated for the blood droplet and smeared blood tests to single out the direct relevance of CAAP to the coagulation cascade in the treatment.

\subsection{Blood-Droplet Tests}

A precipitation process is then applied to separate whole blood into blood plasma and formed elements, which are used to prepare droplet samples in the tests. Shown in row 1 of Figure 1 are the untreated droplets set on glass slides, used as the controls of (a) whole blood, (b) whole blood, (c) blood plasma, and (d) formed elements droplet-samples. The exposure distance and time were $40 \mathrm{~mm}$ and $16 \mathrm{~s}$, respectively. The first whole blood sample in (a) was treated by the hot airflow of a hair dryer, which raised the sample temperature to about $61^{\circ} \mathrm{C}$, which is higher than that of about $47^{\circ} \mathrm{C}$ raised by the plasma flow. A photo of the sample taken after this hot air treatment is presented in row 2 of Figure 1(a) for a comparison. The airflow deformed the shape of the blood droplet, but it didn't instigate any blood clotting.

The other three controls in row 1 of Figures 1 (b)-(d) were treated by the CAAP. The test results are presented in row 2 of Figures 1 (b)-(d) for treated (b) whole blood, (c) blood plasma, and (d) formed elements samples. A dark brown shell, manifesting blood clotting, can be seen in the whole blood and formed elements samples but is not observed in the treated blood plasma sample. The treated formed elements sample is darker, dryer, and harder than the treated whole blood sample. The observation evidences that the clotting of the treated

(1)

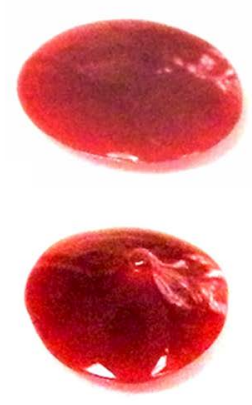

(a)

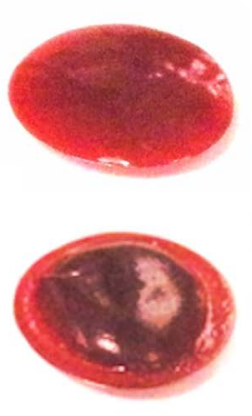

(b)

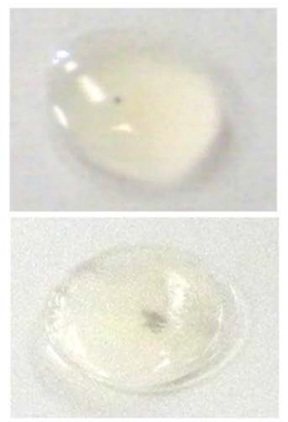

(c)

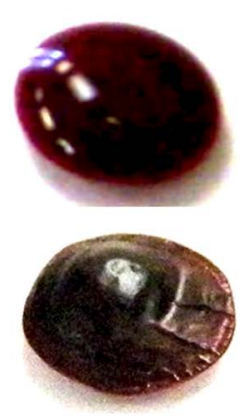

(d)

Figure 1. Untreated controls (row 1) of whole blood (1a), whole blood (1b), blood plasma (1c), and formed elements (1d) droplet-samples; and the corresponding hot air treated sample (2a) and plasma treated samples ( $2 \mathrm{~b}$ to $2 \mathrm{~d}$ ). 
samples in (b) and (d) is ascribed to the activation of the formed elements by the reactive species in the plasma effluent. The results clearly demonstrate the coagulation efficacy of the CAAP employed in the tests, which shortens the clotting time from 25 minutes margin to 16 seconds margin.

Heat can induce protein coagulation; on the other hand, there is no indication of clotting can be found in the treated blood plasma droplet sample (row 2 of Figure 1(c)). It suggests that the heat delivered by the CAAP cannot be a cause of blood clotting, shown in row 2 of Figure 1(b) and Figure 1(d), in the CAAP treatment. This is further evidenced by the comparison of (a) and (b) in row 2 of Figure 1, which rules out the function of the plasma effluent heating to the forming of the clot appearing in (b).

CAAP treatments with different exposure distance and time on each blood droplet sample, which was set on a glass slide, were performed to correlate the OI flux with the degree of clotting. The total amount of OI applied to the blood droplet sample in treatment will be proportional to the total exposure time and inversely proportional to the exposure distance. Three samples treated at the same exposure distance of $25 \mathrm{~mm}$ for 8,12 , and $16 \mathrm{sec}$, respectively, are presented in Figures 2(a)-(c) for comparison. The sample temperatures were less than $53^{\circ} \mathrm{C}$. A shell, formed on each blood sample surface, can be clearly seen. The photos indicate that the degree of blood clotting increases with the increase of the exposure time from 8 to $16 \mathrm{sec}$. Three samples treated at three different exposure distances of 25,30, and $40 \mathrm{~mm}$ for the same exposure time of $16 \mathrm{sec}$ are presented in Figures 2(c)-(e). Again, the sample temperatures were less than $53^{\circ} \mathrm{C}$. As shown, the degree of blood clotting decreases as the exposure distance increases from 25 to $40 \mathrm{~mm}$.

\subsection{Tests on Smeared Blood Sample}

Untreated (control) and CAAP-treated smeared whole blood and formed elements samples were prepared for cell staining and microscopy analysis, which

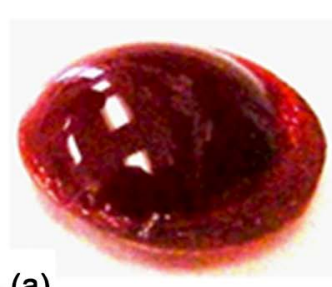

(a)

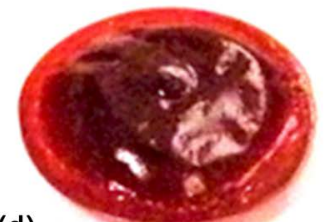

(d)

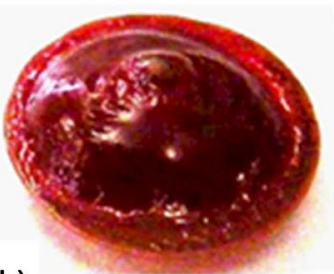

(b)

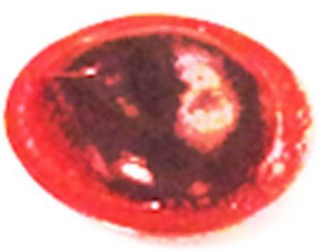

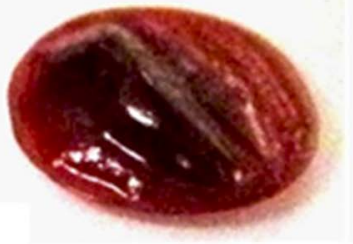

(c)

Figure 2. Blood samples treated by the CAAP at a fixed exposure distance of $25 \mathrm{~mm}$ with three exposure times of (a) $8 \mathrm{sec}$, (b) $12 \mathrm{sec}$, and (c) $16 \mathrm{sec}$; and by the CAAP with a fixed exposure time of $16 \mathrm{sec}$ at two increased exposure distances of (d) $30 \mathrm{~mm}$ and (e) $40 \mathrm{~mm}$. 
identified cell types and performed cell counts. Presented in Figure 3 are the results of RBC and platelet cell counts, from smeared whole blood control and from treated samples with four different exposure times of 2, 3, 4, and $10 \mathrm{~s}$ at 25 $\mathrm{mm}$ exposure distance; and from a smeared whole blood sample treated at 40 $\mathrm{mm}$ exposure distance with 10s exposure time; and from smeared formed elements control and from treated sample with exposure time of $16 \mathrm{~s}$ and exposure distance at $30 \mathrm{~mm}$.

As shown, the smeared whole blood samples treated at a fixed exposure distance of $25 \mathrm{~mm}$, the concentration of RBC/platelets increases/decreases monotonically as the exposure time increases. Cell counts of RBC and platelets with 10 s exposure time at $25 \mathrm{~mm}$ and $40 \mathrm{~mm}$ exposure distances were compared. As shown, the treatment at longer exposure distance causes less changes on the concentrations of $\mathrm{RBC}$ and platelets. The concentration of $\mathrm{RBC} /$ platelets of the smeared formed elements samples also increases/decreases with the CAAP treatment. Experimental results show that CAAP activates RBC and platelets; increase of the applied OI causes the reduction of the platelet count (i.e., increase of activated platelet count) and the increase of RBC count in the treated sample.

The results of the tests on blood droplet samples presented in Figure 2 and the microscopy analysis of smeared whole blood/formed elements samples presented in Figure 3 evidence that the rising of coagulation with plasma treatment correlates with the increasing of RBC and decreasing of platelet counts. The observations are also correlated to the increase of applied OI in the treatment. It advocates that OI carried by the plasma effluent could rapidly trigger coagulation cascade.

When interacted with $\mathrm{H}_{2} \mathrm{O}$, atomic oxygen (OI) can generate large amount of reactive oxygen species (such as $\mathrm{H}_{2} \mathrm{O}_{2}$ and $\mathrm{OH}$ ), which are expected to function similarly to those oxidants, produced or released in the vascular lumen. Several key steps in coagulation cascade are then triggered by the oxidants. They stimulate

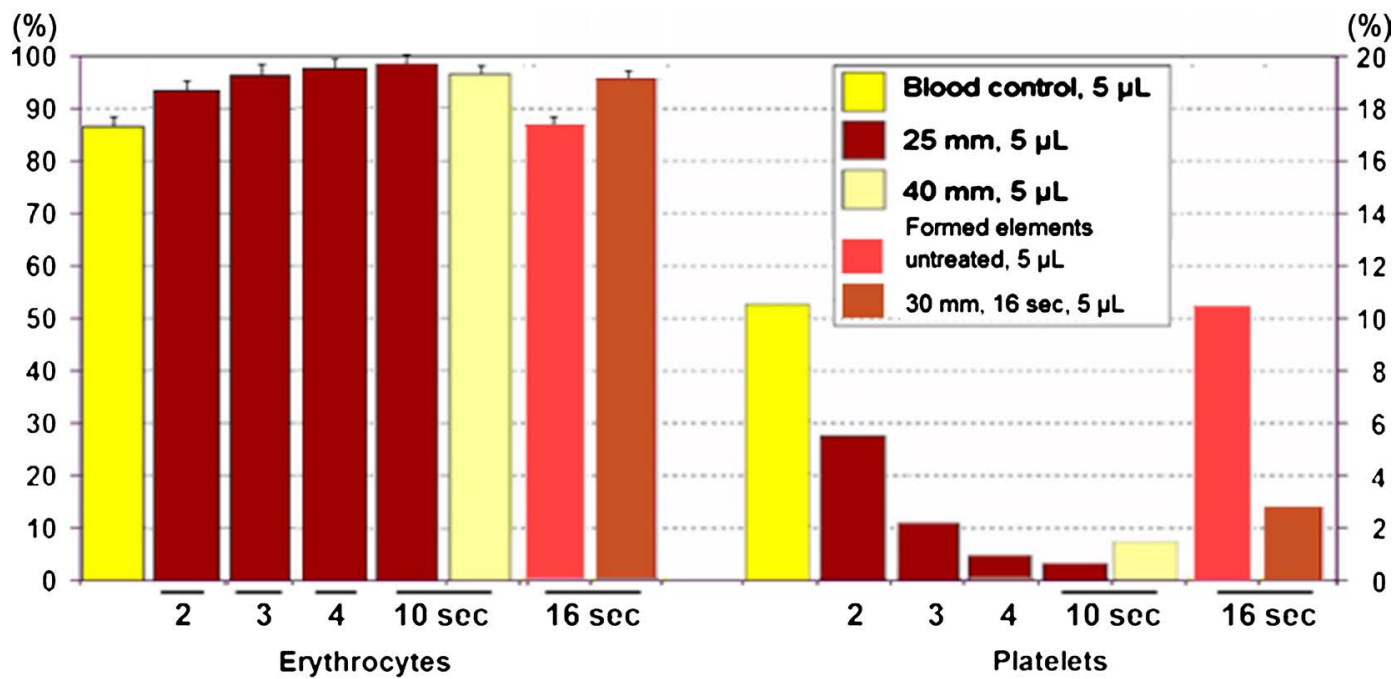

Figure 3. Changes of the RBC and platelet concentrations of smeared whole blood/formed elements samples with the exposure time and with the exposure distance. 
RBC-platelets interaction, which moves the concentration of cells suspended in blood; the observations of the microscope presented in Figure 3 show the increase of RBC concentration in line with the increase of the exposure time. Enhanced adenine nucleotides released by aggregated RBC trigger platelet adherence/agglomeration [19] [21]-[26], where fibrinogen acts as a bridge to link activated platelets together. Subsequently, globular complexes are formed to trap RBC and platelets; the viscosity of blood will also be affected by oxidants that presumably contribute to the denaturation of albumin [20] as well as other proteins found in the blood. Consequently, the blood fluid identity decreases and coagulation rises. Additionally, platelets are activated by oxidants to release the contents of stored granules into the blood plasma to induce thrombin, which converts the soluble fibrinogen, large and complex glycoprotein, into insoluble fibrin strands to form fibrin gel holding activated platelets to form hemostatic plug.

\subsection{A Plausible Mechanism}

In the air discharge, where oxygen has lower ionization energy than that of the nitrogen, electrons $\left(\mathrm{e}^{-}\right)$and molecular oxygen ions $\left(\mathrm{O}_{2}^{+}\right)$are produced. As the air plasma flows out of the discharge zone, atomic oxygen (OI) is generated in the plasma effluent via a dissociative recombination process $\mathrm{O}_{2}^{+}+\mathrm{e}^{-} \rightarrow \mathrm{O}+$ $\mathrm{O}$ as well as via other processes.

Atomic oxygen is very reactive and can oxide most elements. It is essential for respiration of animals and can be used in medical applications. It kills disease microorganisms and improves cellular function. The test results further reveal its significant function of promoting hemostasis.

Cartoon plots presented in Figure 4 incorporate the correlations deduced from the test results, which include the dependencies of the degree of clotting, the RBC count, and the platelet count, on the amount of atomic oxygen supplied in the CAAP treatment. The sequence of the plots illustrates a plasma clotting mechanism described in the following.

Several key steps in the coagulation cascade are triggered by the atomic oxygen, which functions similarly to the oxidants produced or released in the vascular lumen. Atomic oxygen and its oxidant products in the blood plasma stimulate RBC-platelets and WBC interactions (Figure 4(a)). The interactions

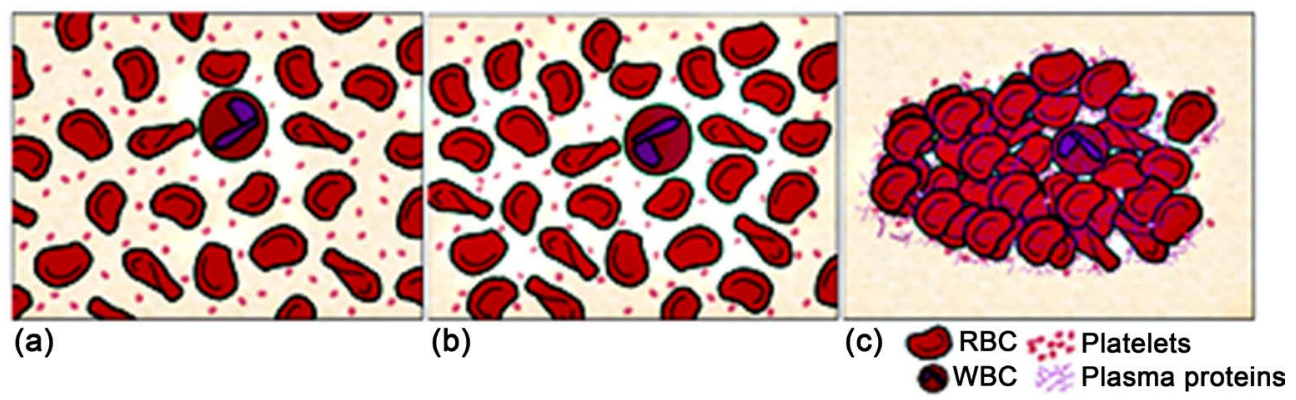

Figure 4. Cartoon plots showing the clotting process in the CAAP treatment. 
influence the concentration of cells suspended in blood (Figure 4(b)). The observations of the microscope presented in Figure 3 show the increase of RBC concentration in line with the increase of the exposure time. Enhanced adenine nucleotides released by aggregated RBC trigger platelet adherence/agglomeration, where fibrinogen acts as a bridge to link activated platelets together. Atomic oxygen also directly activate and aggregate platelets by inactivating anticoagulation agents, such as endothelial-derived relaxing factor (EDRF) which counteracts platelet aggregation to control clotting. Subsequently, globular complexes (Figure 4(a) and Figure 4(b)) are formed to trap RBC and activated platelets, leading to the decrease of inactivated platelet concentration; the viscosity of blood samples will also be affected by atomic oxygen and oxidants that presumably contribute to the denaturation of albumin as well as other proteins found in the blood. Consequently, the blood fluid identity decreases and coagulation rises, leading to the formation of platelet clot (Figure 4(c)).

The critical role of atomic oxygen/ROS in enhancing coagulation can be further justified by comparing the coagulation time of cold atmospheric plasma (CAP) treatment by different plasma devices employing different working gases. The kINPen Plasma MED [6] is an atmospheric pressure plasma jet (APPJ) using Argon gas as the working gas. It generates ROS only after encountering the ambient air outside the generator. It takes 10 minutes to achieve a complete clot. On the other hand, CAAP treatment reaches a complete clot in 16 seconds. In addition, the air plasma of a DBD device can also initiate noticeable partial coagulation in 15 seconds [7].

Because such in Vitro tests do not involve complete coagulation cascade and processes, the test results cannot fully reveal the CAAP-efficacy on hemostasis; in Vivo tests are necessary and are presented in the next Section.

\section{In-Vivo Test to Verify CAAP Hemostasis Efficacy}

In the following, test and the result are presented. An adult swine with blood pressure compatible to that of an adult human being was used as an animal model, and the trial was repeated once. This swine was first injected with calmative-Stresnil and fastened on a table. The swine was then anesthetized with Isoflurance-Fluothane which kept it in a narcotized state, which was monitored throughout the entire trial period.

Shown in Figure 5(a) is a large and deep cut wound on the back of a swine. One artery was cut to cause main bleeding. Because tourniquet couldn't be applied, one had to apply pressure in front of the wound to slowdown active hemorrhage. Thus, the first step was clearing away the blood with medical cloth to locate the cut artery, and then used a finger to press the artery above the cut location as indicated in Figure 5(a). A hand-held CAAP was applied to first treat this cut artery as shown in Figure 5(b). After this artery was sealed, i.e., the bleeding was stopped, CAAP was then swept back and forth to treat other cut area, as shown in Figure 5(c). The total treatment time was about $25 \mathrm{~s}$. 


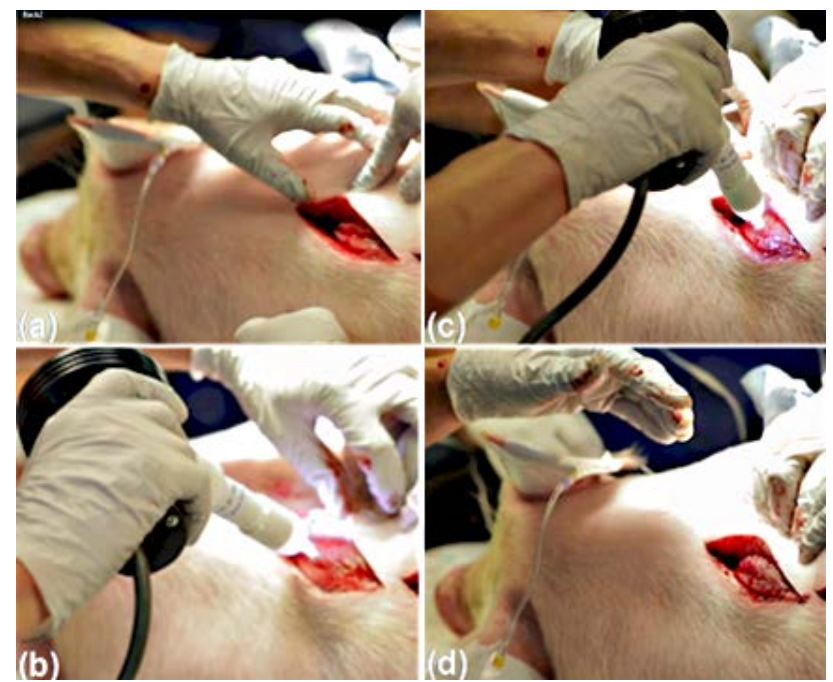

Figure 5. CAAP treatment on a cut wound on the back; (a) inspecting the wound and pressing the cut artery to slow down bleeding, (b) treating the cut artery first, (c) sweeping CAAP back and forth to treat other area of the cut, and (d) assessing the wound to confirm complete hemostasis.

Subsequently, the wound was examined if hemorrhage was controlled. As shown in Figure 5(d), all hemorrhage was stopped. Because the cut artery has relatively small size, it took very short time $(<25 \mathrm{~s})$ to seal it. At 30 minutes later, the wound was check again; it confirmed that there was no re-bleed.

In the future plan, different kinds of wounds will be tested on swine. The results of successful tests can be applied for developing a CAAP based first aid device and its operation protocol in treatment of different wounds. If the clinic trials are still necessary, these results will be useful reference.

\section{CAAP Hemostasis Mechanism}

The in-vivo test result presented in Sec. 3 clearly shows that this CAAP spray rapidly stops hemorrhage from a large cut wound. In Sec. 2, atomic oxygen produced in the plasma effluent is identified to be the dominant factor instigating coagulation cascade.

Hemostasis [27] comprises three major events that occur in a set order following the loss of vascular integrity:

1) Vascular constriction and blood viscosity elevation to slow down bleeding.

2) Platelets are activated by thrombin and change their shape. The protein fibrinogen aggregates platelets and stimulates platelet clumping by binding to collagen at the site of injury, forming a temporary, loose platelet plug.

3) A fibrin mesh (also called the clot) forms and entraps the plug.

In the treatment, the atomic oxygen (OI) delivered by the CAAP interacts with water $\left(\mathrm{H}_{2} \mathrm{O}\right)$ content in the blood, oxidants are produced. Oxidants alter tissue factor pathway inhibitor (TFPI) structure that a pro-coagulant state in endothelial cells is induced. They neutralize nitric oxide (NO) to reverse vasodilator activity and regulate plasminogen-induced IL- $1 \beta$ and TNF- $\alpha$ production in 
microglia to degrade plasmin in anticoagulation. The viscosity of blood is affected by oxidants that presumably contribute to the denaturation of albumin and other proteins found in the blood. Consequently, the blood fluid identity decreases to slow down bleeding. These oxidants also target platelets, in the same way as those produced or released in the vascular lumen, to affect several key steps of platelet function, such as platelet activation, aggregation, and adhesion.

The activated platelets release the contents of stored granules into the blood plasma and change shape from spherical to stellate; and the fibrinogen cross-links with platelets' glycoprotein to aggregate adjacent platelets, which are then bound to the collagen exposed on endothelial cell surfaces. A glycoprotein called von Willebrand factor (vWF), which is found in blood plasma, further strengthens this adhesion by binding collagen to the platelets; this binding activates platelet integrin, which mediate tight binding of platelets to the extracellular matrix and thus adhere this platelet plug (white clot) to the site of injury; this "primary hemostasis" process is accelerated significantly by the OI flux delivered by the CAAP.

$\mathrm{H}_{2} \mathrm{O}_{2}$, produced by OI, stimulates phospholipase A2 enzyme to amplify platelet response to collagen and acts as second messenger by activating arachidonic acid metabolism and phospholipase C (PLC) pathway [28]. It rapidly advances platelet aggregation to set off an increase in the release of $\mathrm{C}_{a}^{2+}$ ions, which, together with thrombokinase/thromboplastin (factor III) released by the damaged tissues, react with prothrombin to form thrombin. Thrombin converts soluble fibrinogen into insoluble fibrin to deposit into the platelet plug and also activates platelets to mediate the formation of covalent bonds, which crosslink the fibrin polymers to form fibrin mesh (red clot) all around the platelet plug to hold it in place. The supplied OI flux also speeds up this "secondary hemostasis" process.

However, the blood pressure of the artery is too high to seal its cut by a blood clot from the outside; thus, in the CAAP treatment, vascular constriction and blood clotting right inside the cut (i.e., internal clot formation) are likely the mechanism of sealing the cut artery of a large cut wound, shown in Figure 5.

In sum, this CAAP is effectual to trigger various biochemical reactions to promote hemostasis. It speeds up vascular constriction, activates platelets, and instigates the crosslinks of various blood polymers to set off coagulation cascade, which boost hemostasis as demonstrated by the test results.

\section{Discussion}

Some injuries can cause a trauma patient to deteriorate rapidly, the time delay between injury and treatment has to be kept to a minimum. It is well established that the patient's chances of survival are the greatest if they receive definitive care within a short period of time after a severe injury [29].

The golden hour represents the span of time in which treatment of bleeding offers the greatest hope of survival [30]. Moreover, it is also called for a full con- 
trol of hemorrhage within as few as 10 minutes (so called platinum ten) to ensuring the survival of wounded persons in accidents.

The first aid [31] of critical traumatic patients in the emergency situation requires effective medical tool, which is capable to stop main hemorrhage of the wound within the initial 10 minutes period and to prevent infection. It is preference that the first aid tool is portable, in particular, in the battlefield and at accident site.

Moreover, the time that has elapsed since the wound occurred is an important factor in determining whether the wound should be closed primarily. Fast bleeding control together with proper disinfection in the treatment will allow some of wounds to be closed quickly without an increased risk of infection; that can reduce the patient's discomfort, speed wound healing, and decrease scarring.

The effectiveness of the CAAP in rapid control of active hemorrhage has been demonstrated (Figure 5). The biocidal effects of the CAAP [13] also provide disinfection simultaneously in the treatment. Further tests on wounds of artery cuts at different locations will be useful to establish the treatment procedure in the first aid in any situation. A CAAP device, which can be made portable by using battery as the power source [32], should meet the requirements of advanced first aid [33]. It is understandable that the required treatment time to stop bleeding varies with the wound. Certain main hemorrhages, such as an amputated leg, will no doubt need much longer treatment time. However, the same CAAP device will be applicable as long as the energy capacity of the battery is able to handle the power demand throughout the running period of the device.

Finally, CAAPs are able to kill bacteria by protein degradation in surface structures and damaging microbial DNA without being harmful to human tissues. Reactive oxygen species (ROS) and other components of plasma affect eukaryotic cells, especially on keratinocytes in terms of viability, proliferation, DNA, adhesion molecules and angiogenesis. Thus, CAAPs can also support wound healing, by its antiseptic effects, by stimulation of proliferation and migration of wound relating skin cells, by activation or inhibition of integrin receptors on the cell surface or by its pro-angiogenic effect. As reported in the reference [13], the postoperative observation of wound healing after CAAP treatment to stop bleeding of a cut to an ear artery and of a cross cut wound in the ham area of a pig model, no any side effects caused by the CAAP treatment were observed. The observation also revealed a positive effect of CAAP treatment on wound healing. It shortened the healing time of the cross cut wound from 14 days to 8 days.

\section{Acknowledgements}

The author is grateful to Alessandro Betti and Edmund Chow for helping me to develop the air plasma generator used in the experiments. This work was supported in part by the Adventix Technologies Inc. and the Aceso Plasma, LLC. 


\section{Conflicts of Interest}

The author declares no conflicts of interest regarding the publication of this paper.

\section{References}

[1] Kuo, S. (2018) Cold Atmospheric Plasmas: Their Use in Biology and Medicine. World Scientific, Singapore.

[2] Stoffels, E., Flikweert, A.J., Stoffels, W.W. and Kroesen, G.M.W. (2002) Plasma Needle: A Non-Destructive Atmospheric Plasma Source for Fine Surface Treatment of (Bio)Materials. Plasma Sources Science and Technology, 11, 383. https://doi.org/10.1088/0963-0252/11/4/304

[3] Kogelschatz, U. (2003) Dielectric-Barrier Discharges: Their History, Discharge Physics and Industrial Applications. Plasma Chemistry and Plasma Processing, 23, 1-46. https://doi.org/10.1023/A:1022470901385

[4] Laroussi, M. (2015) Low Temperature Plasma Jet for Biomedical Applications: A Review. IEEE Transactions on Plasma Science, 43, 703-711. https://doi.org/10.1109/TPS.2015.2403307

[5] Laroussi, M. (2002) Non-Thermal Decontamination of Biological Media by Atmospheric Pressure Plasmas: Review, Analysis, and Prospects. IEEE Transactions on Plasma Science, 30, 1409-1415. https://doi.org/10.1109/TPS.2002.804220

[6] Bekeschus, S., Schmidt, A., Weltmann, K.D. and Woedtke, T. (2016) The Plasma Jet kINPen-A Powerful Tool for Wound Healing. Clinical Plasma Medicine, 4, 19-28. https://doi.org/10.1016/j.cpme.2016.01.001

[7] Fridman, G., Peddinghaus, M., Balasubramanian, M., Ayan, H., Fridman, A., Gutsol, A. and Brooks, A. (2006) Blood Coagulation and Living Tissue Sterilization by Floating-Electrode Dielectric Barrier Discharge in Air. Plasma Chemistry and Plasma Processing, 26, 425-442. https://doi.org/10.1007/s11090-006-9024-4

[8] Fridman, G., Brooks, A.D., Balasubramanian, M., Fridman, A., Gutsol, A., Vasilets, V.N., Halim, A. and Friedman, G. (2007) Comparison of Direct and Indirect Effects of Non-Thermal Atmospheric-Pressure Plasma on Bacteria. Plasma Processes and Polymers, 4, 370-375. https://doi.org/10.1002/ppap.200600217

[9] Lai, W., Lai, H., Kuo, S.P., Tarasenko, O. and Levon, K. (2005) Decontamination of Biological Warfare Agents by a Microwave Plasma Torch. Physics of Plasmas, 12, Article ID: 023501. https://doi.org/10.1063/1.1843131

[10] Chen, C.Y., Fan, H.W., Kuo, S.P., Chang, J., Pedersen, T., Mills, T. and Huang, C.C. (2009) Blood Clotting by Low Temperature Air Plasma. IEEE Transaction on Plasma Science, 37, 993-999. https://doi.org/10.1109/TPS.2009.2016344

[11] Kuo, S.P., Tarasenko, O., et al. (2009) Contribution of a Portable Air Plasma Torch to Rapid Blood Coagulation as a Method of Preventing Bleeding. New Journal of Physics, 11, Article ID: 115016.

[12] Kuo, S.P., Chen, C.Y., Lin, C.S. and Chiang, S.H. (2010) Wound Bleeding Control by Low Temperature Air Plasma. IEEE Transaction on Plasma Science, 38, 1908-1914. https://doi.org/10.1109/TPS.2010.2047028

[13] Kuo, S.P., Chen, C.Y., Lin, C.S. and Chiang, S.H. (2012) Applications of Air Plasma for Wound Bleeding Control and Healing. IEEE Transaction on Plasma Science, 40, 1117-1123. https://doi.org/10.1109/TPS.2012.2184142

[14] Duarte, S., Kuo, S.P., Murata, R.M., Chen, C.Y., Saxena, D., Huang, K.J. and Popovic, 
S. (2011) Air Plasma Effect on Dental Disinfection. Physics of Plasmas, 18, Article ID: 073503.

[15] Kuo, S.P. (2012) Air Plasma for Medical Applications. Journal of Biomedical Science and Engineering, 5, 481-495. http://www.SciRP.org/journal/jbise/ https://doi.org/10.4236/jbise.2012.59061

[16] Kuo, S.P. (2010) Portable Plasma Sterilizer. US Patent No. 7777151 B2.

[17] Born, G.V.R., Bergquist, D. and Arfors, K.E. (1976) Evidence for Inhibition of Platelet Activation in Blood by a Drug Effect on Erythrocytes. Nature, 259, 233-235. https://doi.org/10.1038/259233a0

[18] Kuo, S.P., Pedersen, T. and Mills, T. (2011) Two-Dimensional Distribution of Atomic Oxygen Multiplet Radiation Produced by an Air Plasma Torch. IEEE Transaction on Plasma Science, 39, 2282-2283. https://doi.org/10.1109/TPS.2011.2155089

[19] Kolev, K. and Colin, L. (2016) Bleeding Related to Disturbed Fibrinolysis. British Journal of Haematology, 1-12. https://doi.org/10.1111/bjh.14255

[20] Meireles, M., Aimar, P. and Sanchez, V. (2004) Albumin Denaturation during U1trafiltration: Effects of Operating Conditions and Consequences on Membrane Fouling. Biotechnology and Bioengineering, 38, 528-534. https://doi.org/10.1002/bit.260380511

[21] Machovich, R. and Owen, W.G. (1990) The Elastase-Mediated Pathway of Fibrinolysis. Blood Coagulation \& Fibrinolysis, 1, 79-90. https://doi.org/10.1097/00001721-199003000-00011

[22] Bergquist, D. and Arfors, K.E. (1980) Haemostatic Platelet Plug Formation in the Isolated Rabbit Mesenteric Preparation-An Analysis of Red Blood Cell Participation. Thrombosis and Haemostasis, 44, 6-8.

[23] Schmid-Schdnbein, H. and Teitel, P. (1979) Basic Aspects of Blood Trauma. Schattauer Verlag, Stuttgart, 322-340.

[24] Tiefenbach, H.J., Durchschlag, H., Schneider, G. and Jaenicke, R. (2004) Thermodynamic Analysis of Serum Albumin Denaturation by Sodium Dodecyl Sulfate. Aqueous Polymer Dispersions, 124, 130-140.

[25] Schmid-Schdnbein, H., Born, G.V.R., Richardson, P.D., Cusack, N., Rieger, H., Forst, R., Rohling-Winkel, J., Blasberg, P. and Wehmeyer, A. (1981) Rheology of Thrombotic Processes in Flow: The Interaction of Erythrocytes and Thrombocytes Subjected to High Flow Forces. Biorheology, 18, 415-444. https://doi.org/10.3233/BIR-1981-183-609

[26] Massberg, S., Grahl, L., von Bruehl, M. and Manukyan, D., et al. (2010) Reciprocal Coupling of Coagulation and Innate Immunity via Neutrophil Serine Proteases. Nature Medicine, 16, 887-896. https://doi.org/10.1038/nm.2184

[27] Boon, B.G. (1993) An Overview of Hemostasis. Toxicologic Pathology, 21, 170-179. https://doi.org/10.1177/019262339302100209

[28] Del Principe, D., Menichelli, A., De Matteis, W., Di Giulio, S., Giordani, M., Savini, I. and Finazzi-Agro, A. (1991) Hydrogen Peroxide Is an Intermediate in the Platelet Activation Cascade Triggered by Collagen, but Not by Thrombin. Thrombosis Research, 62, 365-375. https://doi.org/10.1016/0049-3848(91)90010-T

[29] American College of Surgeons (2008) Advanced Trauma Life Support. American College of Surgeons.

[30] Lerner, E.B. and Moscati, R.M. (2001) The Golden Hour: Scientific Fact or Medical "Urban Legend"? Academic Emergency Medicine, 8, 758-760. 
https://doi.org/10.1111/j.1553-2712.2001.tb00201.x

[31] Jevon, P. and Cooper, L. (2005) First Aid Part 5-Treatment for Severe Bleeding. Nursing Times, 104, 26-27.

[32] Kuo, S.P. (2015) Battery Powered Handheld Air Plasma Spray. US Patent No. 8927896 B2.

[33] Spencer, K. (2016) Air Plasma Spray for First Aid. Open Journal of Emergency Medicine, 4, 69-82. https://doi.org/10.4236/ojem.2016.43010 